\title{
NOTE ON AERODYNAMIC HEATING WITH A VARIABLE SURFACE TEMPERATURE*
}

\author{
By A. E. BRYSON (Hughes Aircraft Company)
}

Emmons [1], has considered the problem of an insulated flat plate of infinite extent started impulsively from rest in a viscous, incompressible fluid. One of the interesting results of his analysis was a simple expression for the temperature recovery factor at the plate surface. Another interesting result can be obtained from the same problem by considering, instead of an insulated plate, a plate with a surface temperature that is a given function of time.

As Emmons has shown, letting $\mu$, the viscosity coefficient of the fluid, $\rho$, the fluid density, and $k$, the thermal conductivity of the fluid, be constant, it follows from the equations of motion and the boundary conditions that the pressure is constant and the velocity normal to the plate is zero. The momentum equations reduce to:

$$
\frac{\partial u}{\partial t}=\nu \frac{\partial^{2} u}{\partial y^{2}}
$$

where $u$ is the velocity component of the fluid parallel to the surface of the plate, $t$ is the time, $y$ is the distance perpendicular to the plate surface, and $\nu=\mu / \rho$. The energy equation becomes:

$$
{ }_{\rho} C_{P} \frac{\partial T}{\partial t}-k \frac{\partial^{2} T}{\partial y^{2}}=\mu\left(\frac{\partial u}{\partial y}\right)^{2}
$$

where $T$ is the fluid temperature and $C p$ is the fluid specific heat per unit mass at constant pressure. These equations are to be solved with the following boundary and initial conditions:

$$
\begin{array}{ll}
u(0, t)=0, & u(y, 0)=U, \\
T(0, t)=T_{s}(t), & T(y, 0)=T_{\infty},
\end{array}
$$

where $U$ is the free stream velocity, $T_{\infty}$ the free stream temperature, and $T_{s}$ the plate surface temperature.

The problem defined by Eqs. (1) and (3) for the velocity diffusion is well-known, the solution being

$$
u=\operatorname{Uerf}\left[y / 2(\nu t)^{1 / 2}\right] .
$$

Substituting (5) into (2), we have

$$
\frac{\partial T}{\partial t}-a \frac{\partial^{2} T}{\partial y^{2}}=\frac{U^{2}}{\pi C p} \frac{\exp \left(-y^{2} / 2 \nu t\right)}{t},
$$

where $a=k / \rho C p$. The solution to (6) with the boundary and initial conditions (4) is obtained by the method of heat sources and sinks and the result is: 


$$
\begin{aligned}
& T-T_{\infty}=2(\pi)^{-1 / 2} \int_{\nu / 2(a t)^{2 / 2}}^{\infty}\left[T_{s}\left(t-y^{2} / 4 a \beta^{2}\right)-T_{\infty}\right] \exp \left(-\beta^{2}\right) d \beta \\
& +\frac{U^{2}}{\pi C p} \int_{0}^{\infty} d \lambda \int_{0}^{t} \frac{\exp \left(-\lambda^{2} / 2 \nu \tau\right)}{\tau} \frac{\exp \left[-\frac{(y-\lambda)^{2}}{4 a(t-\tau)}\right]-\exp \left[-\frac{(y+\lambda)^{2}}{4 a(t-\tau)}\right]}{[4 a \pi(t-\tau)]^{1 / 2}} d \tau .
\end{aligned}
$$

The heat flow through a unit surface of the flat plate, $Q$, is given by the derivative of (7) evaluated at the surface of the plate:

$$
\begin{aligned}
Q=\left.k \frac{\partial T}{\partial y}\right|_{\nu=0}=-k(\pi a t)^{-1 / 2}\left[T_{s}(0)-T_{\infty}\right] & -k(\pi a)^{-1 / 2} \int_{0}^{t}(t-\tau)^{-1 / 2} T_{s}^{\prime}(\tau) d \tau \\
& +k(\pi a t)^{-1 / 2} r(\sigma) U^{2} / 2 C p,
\end{aligned}
$$

where the recovery factor, $r(\sigma)$, is given by:

$$
r(\sigma)=\frac{4}{\pi}\left(\frac{\sigma / 2}{|1-\sigma / 2|}\right)^{1 / 2} \begin{cases}\tan ^{-1}\left(\frac{1-\sigma / 2}{\sigma / 2}\right)^{1 / 2} ; & \sigma<2 \\ \log \left[(\sigma / 2)^{1 / 2}+(\sigma / 2-1)^{1 / 2}\right] ; & \sigma>2\end{cases}
$$

and $\sigma=\nu / a=$ Prandtl number. Equation (8) can be written more compactly in the form of a Stieltjes integral as follows:

$$
Q=-k(\pi a t)^{-1 / 2} \int_{0}^{t}(1-\tau / t)^{-1 / 2} d\left[T_{s}(\tau)-T_{c}\right],
$$

where

$$
T_{e}=T_{\infty}+r(\sigma) U^{2} / 2 C p .
$$

The surface-temperature variation to give a prescribed heat flow variation can be obtained by inverting the Abel integral Eq. (10); this gives

$$
T_{e}-T_{s}(t)=(a / \pi)^{1 / 2} k^{-1} \int_{0}^{t}(t-\tau)^{-1 / 2} Q(\tau) d \tau .
$$

For a constant rate of heat flow this reduces to

$$
T_{e}-T_{s}(t)=2 Q k^{-1}(a t / \pi)^{1 / 2} .
$$

Emmons has already given this latter solution for constant heat flow to the plate [1].

By replacing $t$ by $x / U$ in (1) and (2), we have the linearized boundary-layer equations for steady, viscous, incompressible flow past a flat plate (sometimes called the Rayleigh equations). The boundary conditions (3) and (4) become

$$
\begin{array}{ll}
u(0, x)=0, & u(y, 0)=U, \\
T(0, x)=T_{s}(x), & T(y, 0)=T_{\infty}
\end{array}
$$

These imply a semi-infinite flat plate with an arbitrary surface temperature $T_{s}(x)$, where $x$ is the distance from the leading edge in the direction of flow. Therefore, an approximation to the heat flow to a semi-infinite flat plate in a steady flow of velocity $U$, 
and with an arbitrary surface-temperature distribution $T_{s}(x)$, can be obtained by replacing $t$ by $x / U$ in Eq. (10); this gives

$$
Q=-\pi^{-1 / 2} k(U / \nu x)^{1 / 2} \sigma^{1 / 2} \int_{0}^{x}(1-\xi / x)^{-1 / 2} d\left[T_{s}(\xi)-T_{e}\right]
$$

which, for constant surface temperature reduces to the familiar form

$$
Q=\pi^{-1 / 2} k(U / \nu x)^{1 / 2} \sigma^{1 / 2}\left(T_{e}-T_{s}\right) .
$$

Lighthill [2] has given an expression for heat transfer to an arbitrary two-dimensional surface in terms of the skin friction and temperature along the surface, by using the Fage and Falkner linear approximation of the boundary-layer profile and neglecting the viscous dissipation terms in the energy equation. For the case of the flat plate, his result is:

$$
Q=-0.339 k(U / \nu x)^{1 / 2} \sigma^{1 / 3} \int_{0}^{x}\left[1-(\xi / x)^{3 / 4}\right]^{-1 / 3} d\left[T_{s}(\xi)-T_{\infty}\right] .
$$

He argued that the effect of viscous dissipation is taken care of by replacing $T_{\infty}$ in the above expression by $T_{\infty}+\sigma \frac{1}{2} U^{2} / 2 C p$, the boundary-layer equilibrium temperature. If, following the suggestion of Lewis and Carrier [3], we replace $U$ by $0.35 U$ to approximate a mean convective velocity in the boundary layer the constant multipliers of Eqs. (15) and (16) are nearly equal.

The differential equations used here and by Emmons in [1] apply to a fictitious fluid of constant pressure and density, but variable temperature. The equations are really of interest only because the compressible fluid boundary layer equations can be reduced to their form by the Von Mises transformation and the assumptions that $\mu$ is proportional to the temperature and $\sigma$ is constant (see for example ref. [4]). If enthalpy is used as the independent variable instead of temperature, no additional assumption need be made on the variation of the specific heat with temperature. Then the only change in the previous differential equations is to replace $y$ by $\eta$ where

$$
\eta=\int_{0}^{\nu} \frac{\rho}{\rho_{\infty}} d y
$$

The expressions for heat transfer rate are unchanged, although as they stand $C_{P}$ must be assumed constant.

\section{BIBLIOGRAPHY}

1. H. W. Emmons, Note on aerodynamic heating, Q. Appl. Math. 8, 402-405 (1951).

2. M. J. Lighthill, Contributions to the theory of heat transfer through a laminar boundary layer, Proc. of Roy. Soc. (A) 202, 359-377 (1950).

3. J. A. Lewis and G. F. Carrier, Some remarks on the flat plate boundary layer, Q. Appl. Math. 7, 228-233 (1949).

4. C. R. Illingworth, Unsteady laminar flow of gas near an infinite flat plate, Proc. Cambridge Phil. Soc. 46, 603-613 (1950). 